\title{
The successful medical management of gastric outflow obstruction associated with the use of non-steroidal anti-inflammatory drugs in the elderly
}

\author{
R.J. Geraghty ${ }^{1}$, D. Black ${ }^{1}$ and S.A. Bruce ${ }^{2}$ \\ ${ }^{1}$ Queen Mary's Hospital, Sidcup, Kent and ${ }^{2}$ St. Helen's Hospital, Hastings, East Sussex, UK
}

\begin{abstract}
Summary: One hundred and nineteen consecutive elderly patients with endoscopically diagnosed peptic ulceration were reviewed. Associated gastric outflow obstruction was present in $10.1 \%$. The presenting clinical features differed significantly from typical younger patients and most (11/12) were taking non-steroidal anti-inflammatory drugs, suggesting a possible role for these agents in the pathogenesis of gastric outflow obstruction. These elderly patients have been successfully managed by medical therapy alone.
\end{abstract}

\section{Introduction}

Patients with benign gastric outflow obstruction complicating peptic ulcer disease are usually male, have a long ulcer history and require surgery even after initial successful medical treatment. ${ }^{1,2}$ More recently endoscopic balloon dilatation has been suggested as an alternative therapeutic approach. ${ }^{3}$ The clinical features and management of gastric outflow obstruction in elderly subjects have not been previously studied in detail. Our report also includes details of the successful medical treatment of 12 elderly patients.

\section{Patients, methods and results}

All upper gastrointestinal endoscopies performed in two medical units for the elderly between October 1987 and December 1989 were reviewed. Of 119 patients ( 73 females) aged over 75 years with peptic ulceration there were 12 cases $(10.1 \%)$ of gastric outflow obstruction. The mean age of these patients was 82 years (range $77-89$ years), 10 were female.

The following endoscopic criteria were required to make the diagnosis: (1) an overnight fasting gastric residue greater than $200 \mathrm{ml}$; (2) a narrowed pylorus or first part of the duodenum impassable

Correspondence: R. Geraghty, M.R.C.P., 21 Friars Road, London E6 1LJ, UK.

Accepted: 8 May 1991.

This work was presented in part at the Spring 1990 meeting of the British Geriatrics Society in Belfast. by a standard $11 \mathrm{~mm}$ endoscope; (3) hyperaemic, oedematous, friable or scarred mucosa at the site of the stricture; (4) evidence of acute or chronic pyloric or duodenal ulceration.

The site of the obstruction was the pylorus in 10 subjects and the first part of the duodenum in the remainder. Benign inflammatory biopsies were universally present with no cases of malignancy. Severe oesophagitis, another recognized feature of gastric outflow obstruction, was also present in all cases. The diagnosis was further confirmed by barium study ( 9 subjects) and ultrasound (1 subject).

Presenting symptoms were of recent onset (mean 46 days, range 2-217 days). Vomiting (75\%), weight loss $(58 \%)$ and abdominal discomfort $(67 \%)$ were the most common (Table I); severe ulcer pain was not a feature. Only $25 \%$ gave a past history of proven peptic ulceration and of these one had been previously successfully treated for gastric outflow obstruction by medical therapy alone and was already taking ranitidine (Table I). Eleven of the 12 had been taking non-steroidal anti-inflammatory drugs (NSAIDs) for a mean of 6 months (range 2 months -2 years).

Relevant physical signs (present in only 5 subjects) were epigastric tenderness and/or a succussion splash.

All responded rapidly to medical treatment alone with complete resolution of presenting symptoms after a mean of 8.3 days (range $2-14$ days). NSAIDs were discontinued in all cases. Intravenous fluids and anti-emetics were given as appropriate. $\mathrm{H}_{2}$-antagonists were prescribed orally in all 


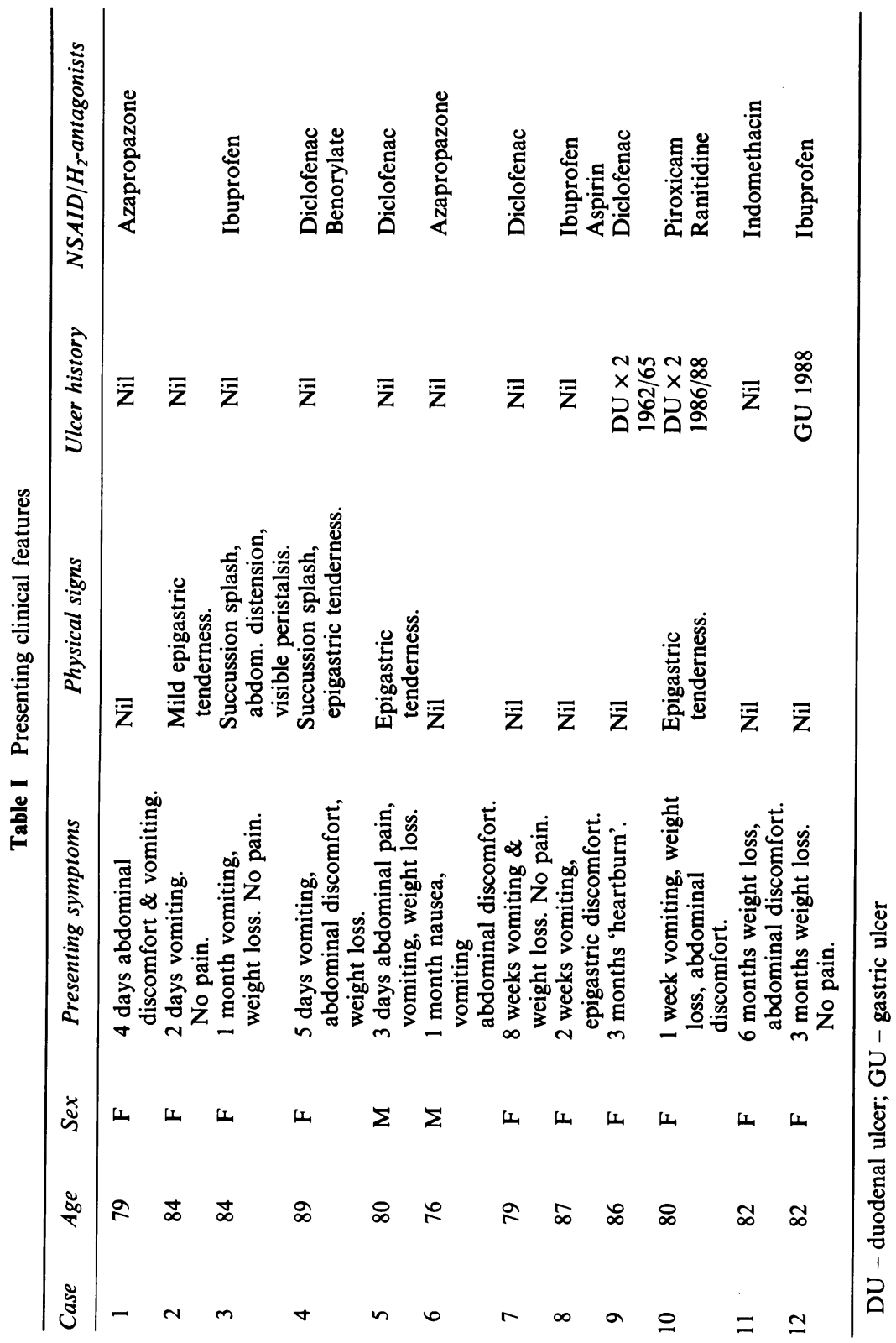


cases. Doses used varied between patients (ranitidine $300-900 \mathrm{mg}$ daily or cimetidine $800-1600 \mathrm{mg}$ daily). In the one patient already taking ranitidine the dose was increased and bismuth chelate (DeNol) added. No recurrence of symptoms was reported with $\mathrm{H}_{2}$-antagonist maintenance therapy (ranitidine $150-300 \mathrm{mg}$ daily or cimetidine $400-$ $800 \mathrm{mg}$ daily). Patients were followed up for a mean of 10 months (range 1.5-24 months). In the 10 subjects for whom records were available, weight gain followed treatment in 8 , including all but one of those presenting with weight loss (Table II). One patient died of an unrelated cause.

\section{Discussion}

Our diagnostic criteria for gastric outflow obstruction are comparable with previous reports, ${ }^{2-4}$ although nuclear medicine studies were not available. In particular, failure to pass an $11 \mathrm{~mm}$ diameter endoscope through the stricture has been used to confirm the diagnosis. ${ }^{3}$

The overall incidence of benign gastric outflow obstruction is declining. This is attributed to the more successful medical treatment of peptic ulcers with the introduction of $\mathrm{H}_{2}$-antagonists. ${ }^{4}$ We have found the incidence in the elderly comparable with younger subjects $(12 \%$ of 738 with peptic ulceration), ${ }^{2}$ but higher than in a previous retrospective surgical study of ulcer disease in the aged which does not provide clinical or diagnostic data for the sub-group with gastric outflow obstruction. ${ }^{5}$

In some respects the clinical features of our subjects differ considerably from typical younger patients who have well documented chronic peptic ulceration with long histories of intermittent ulcer pain. In such cases the development of gastric outflow obstruction has been heralded by persistent and worsening ulcer pain, weight loss and finally vomiting. In contrast, gastric outflow obstruction was the presenting feature of peptic ulceration in most of our patients. The absence of

\section{References}

1. Hermann, R.E. Obstructing duodenal ulcer. Surg Clin North Am 1976, 56: 1403-1411.

2. Welland, D., Dunn, D.H., Humphrey, E.W. \& Schwartz, M.L. Gastric outlet obstruction in peptic ulcer disease: an indication for surgery. Am J Surg 1982, 143: 90-93.

3. Craig, P.I. \& Gillespie, P.E. Through the endoscope balloon dilatation of benign gastric outlet obstruction. Br Med J 1988, 297: 396

4. Ellis, H. Pyloric stenosis complicating duodenal ulceration. World J Surg 1987, 11: 315-318.

5. Watson, R.J., Hooper, T.L. \& Ingram, G. Duodenal ulcer disease in the elderly: a retrospective study. Age Ageing 1985, 14: 225-229. physical signs, however, is not unusual. ${ }^{1,2}$ Although peptic ulceration was the underlying cause of gastric outflow obstruction in all our patients, ulcer pain is often absent in the elderly ${ }^{6}$ and may have been further masked by the use of NSAIDs thus delaying presentation. ${ }^{7}$

Our success with medical management alone contrasts with the findings in younger patients where it is reported that up to $94 \%$ with duodenal ulcer disease and outflow obstruction ultimately require surgery despite intensive medical therapy including cimetidine, ${ }^{2}$ and with older patients in whom $87.5 \%$ are reported to require surgery. ${ }^{5}$ Our mean response time was 8.3 days. This is noteworthy as the standard criteria for surgery has been failure to respond to intensive medical therapy within 5 days. $^{2}$

Diminished levels of prostaglandin $E_{2}$ have recently been implicated in the pathogenesis of gastric outflow obstruction associated with pyloric oedema and scarring in younger patients. ${ }^{8}$ This suggests a possible mechanism whereby NSAIDs might predispose to gastric outflow obstruction and we believe it should be added to the list of possible NSAID-induced gastrointestinal complications in the elderly.

Our success with medical treatment may be explained by a predominant element of pyloric spasm and oedema induced by acute ulceration and NSAIDs. However, even in this clinical context, over $60 \%$ of younger patients have required surgery. ${ }^{2}$ We believe weight gain, as documented in most subjects, to be an important marker of the successful treatment of peptic ulcer disease.

This series suggests that, in contrast to typical younger patients with gastric outflow obstruction who are male, have longstanding and worsening ulcer symptoms ${ }^{1}$ and require surgery, ${ }^{2}$ the elderly are predominantly females taking NSAIDs who present acutely and ultimately respond to medical management alone although this may take longer than the conventional 5 days.

6. Clinch, D., Banerjee, A.K. \& Ostick, G. Absence of abdominal pain in elderly patients with peptic ulcer. Age Ageing 1984, 13: 120-123.

7. Skander, M.P. \& Ryan, F.P. Non-steroidal anti-inflammatory drugs and pain free peptic ulceration in the elderly. $\mathrm{Br}$ Med $\mathrm{J}$ 1988, 297: 833-834.

8. Goldman, G., Tiomny, E., Kahn, P.J. et al. Prostaglandin $E_{2}$ in pyloric stenosis. Arch Surg 1989, 124: 724-726. 